\title{
Improving Social Media Monitoring and Analysis Tools for Emergency Management
}

\author{
Claire Mason and Robert Power \\ Commonwealth Scientific and Industrial Research Organisation \\ Email: robert.power@csiro.au
}

\begin{abstract}
Open source data from social media platforms is becoming a critical source of intelligence to inform situational awareness in the emergency management domain. However, the use of social media data in emergency management organisations is not yet widespread. This paper reports on findings from four interviews with social media subject matter experts from Australian emergency management organisations. The first goal of the interviews was to understand how social media monitoring and analysis tools are used to meet the information needs of emergency services organisations. A second goal of the interviews was to capture their insight as to how such tools could be improved, to inform the ongoing development of CSIRO's Emergency Situation Awareness (ESA) tool. The ESA tool provides crowd sourced information in near-realtime from Twitter about all-hazard types for emergency managers. ESA collects Tweets from Australia and New Zealand and processes them to: identify unexpected incidents, monitor ongoing emergency events and provide access to an archive to explore past events (Power et al. 2014).

This paper reports on the findings from these interviews. All four interviewees were using a range of social media monitoring and analysis tools such as Geofeedia and Signal as well as the ESA tool. Our analysis revealed that participants valued the following characteristics when analysing social media data for emergency management: saving time, triangulation of multiple sources of information, "More eyes on the ground", geographic and temporal context, information about individuals, collaborative tools and cost minimisation.
\end{abstract}

However, as one participant noted: "there is no one product that suits all our needs". We spoke to participants about how the current social media monitoring and analysis tools could be improved. The key features they sought were:

1. Automated evaluation of information sources

2. The ability to follow social media content from specific individuals

3. Geographic representation of search results

4. Access to real-time feeds across multiple platforms, and

5. Flexibility to retrospectively modify searches.

We conclude by describing how these recommendations have been used to improve the delivery of CSIRO's ESA tool.

Keywords: $\quad$ Emergency management, situational awareness, social media, Twitter 


\section{INTRODUCTION}

Emergency management organisations draw upon a wide range of data to inform their situational awareness, ranging from informants, media, sensors, communications tools and maps. Open source data from social media platforms is becoming a complementary and critical source of intelligence to support emergency preparedness, response and recovery (DHS 2014). However, it remains a fairly specialised domain. Tapia et al. (2013) focused on the use of social media data in large international disaster response organisations. They found a varied uptake with pockets of use and acceptance among organisations. Plotnik et al. (2015) surveyed 241 U.S. emergency managers at the county level and found that only about half use social media in any way. Most do not have any formal policies to guide their use, but of those that do, about one quarter forbid the use of social media. Similarly, Lindsay (2011) reported that the use of social media is yet to be fully utilised. They found that during crisis events, the emergency services effectively use social media to provide information to the community, but their ability to obtain information from the public is limited.

Although the use of social media monitoring and analysis to inform emergency management is not yet widespread, there are now specialists with considerable expertise in this space. The goal of our research was to engage with these specialists to better understand how social media monitoring and analysis tools are currently being used and how such tools can be improved to better meet the information needs and work flows of emergency management organisations.

\section{APPROACH}

CSIRO developers of the ESA tool were already in contact with key emergency management organisations in several states of Australia. From this network they identified ten subject matter experts in the use of social media to inform emergency management. Three initial contacts from different states and in different types of emergency management organisations were chosen so as to provide a range of perspectives for the research.

The three contacts were sent an email inviting them to take part in an interview with two members of the research team. In the email, the purpose of the interviews was described as follows:

These interviews form part of a research project that CSIRO is carrying out to understand how information captured from social media analysis and monitoring tools can inform emergency management in Australia. This research will be used to inform CSIRO's digital productivity research and the development of their Emergency Situation Analysis tool.

Two participants accepted the interview request directly. The third nominated two other individuals to participate instead, who agreed to take part in an interview. In Table 1 below we list the participant position titles along with a short description of the role played by their organisation.

Table 1. Participants' roles in the Emergency Management domain.

\begin{tabular}{|l|l|}
\hline \multicolumn{1}{|c|}{ Position title } & \multicolumn{1}{c|}{ Organisation's role } \\
\hline Manager, Operational Intelligence & $\begin{array}{l}\text { State-level coordination of emergency management } \\
\text { organisations }\end{array}$ \\
\hline $\begin{array}{l}\text { Section Leader, Earthquake Alert and Tsunami } \\
\text { Warning Section }\end{array}$ & $\begin{array}{l}\text { National and international monitoring of earthquakes } \\
\text { and tsunamis }\end{array}$ \\
\hline Manager Open Source Intelligence Capability & State Police service \\
\hline Team Leader, Intelligence Unit & State Police service \\
\hline
\end{tabular}

The interviews were conducted face-to-face at the interviewee's place of work and all lasted at least one hour. The longest took two hours and forty-six minutes. They were conducted in a semi-structured manner and all were recorded with participants' permission. The key questions from the interviews were as follows:

1. What are the key roles in your work area?

2. What social media monitoring and analytics tools do you currently use in your work area?

3. What do these tools do well and not well in terms of your organisation's information needs?

4. How could these tools be improved to better meet your organisation's information needs?

Both members of the research team took extensive notes from the interviews. Recordings were used to capture additional detail and quotes for illustration. However, the researchers' notes represented the primary source of data for this report. The findings are presented in Sections 2 and 3 below and focus on our original areas of interest: how social media is currently used in these organisations and what improvements to the tools can be made. 


\section{THE ROLE OF SOCIAL MEDIA TOOLS IN EMERGENCY MANAGEMENT}

All interview participants described social media data as a source of intelligence that served to improve situational awareness about potential or identified emergency response events. This intelligence was used in different ways during different phases of the emergency management work cycle. Outside of an emergency event, social media monitoring and analysis has a more general focus, being used as one of several sources of information to monitor and scan the environment. However, when an event (or potential event) is identified, it is used in a more targeted way, to understand the "five w's" of "who", "what", "when", "where" and "why" and to capture more detailed information. To this end, they all monitored, collated and analysed multiple sources of data and then integrated it so as to inform key stakeholders and decision-makers in the emergency management space.

The nature of this role could be seen to influence the way in which they used social media monitoring and analysis and the characteristics that they sought from these tools, namely: the ability to save time, triangulation of multiple sources of information, achieve "more eyes on the ground", contextualise data geographically and temporally, capture information about individuals, support collaboration and minimise costs. Below, we outline why each of these factors was seen to be important.

\subsection{Saving Time}

Time urgency is inherent to an emergency situation. For an intelligence officer, this plays out in several ways. First, they value the immediacy of the information provided via social media platforms because it can be used to identify a potential emergency event faster and thus prepare the relevant actors earlier (emergency services, relevant ministers). Two of our participants gave examples in which the information derived from social media monitoring and analysis tools had alerted them to an event more quickly than traditional intelligence channels:

We had an incident with a gunman ... it had already started to pick up on social media and ....it was picked up on ESA before people had actually reported it to police. A lot of the younger generation their first instinct is to capture it and speak about it on social media rather than to notify... have a policing response [Interviewee 3]

For events near the population... Twitter assists... giving us a heads up in some instances, saying something is going on. And then you see the seismic waves coming in. [Interviewee 2]

While time urgency gives value to the real-time information provided on social media channels, it also poses a challenge. An enormous volume of information is disseminated via social media, creating a major challenge for intelligence offers who need to be able to filter out irrelevant or inaccurate information so that relevant information can be identified in a timely manner. Consequently, the quality of the filtering tools was extremely important. These tools need to be able to capture a large amount of information, accurately filter out irrelevant information and provide results in a timely manner. Our participants valued software that allowed them to construct searches with keywords and filters. In addition, when social media monitoring is being used to identify a potential emergency event, analysis tools should provide an immediate alert if there are signs that an event may have occurred (e.g., a bushfire has started, an earthquake felt, a protest is gathering). In this regard, the ESA tag cloud was seen to be particularly useful, since it is configured to continuously monitor tweets in real-time, constantly updating a word cloud which represented the most frequently used words and triggering email alerts if relevant terms (e.g., "smoke", "fire", "earthquake") were being used at a higher level than normal. Tools which required a longer time to generate or comprehend search results (e.g., 12 hours) were not useful because they didn't provide the real-time intelligence needed to inform emergency response teams.

The ability to save time was also important in terms of being able to drill down into the detail of the results. When intelligence officers received search query results of interest, they needed to be able to examine the findings in more detail so as to verify their accuracy and gain a more detailed understanding. Therefore, participants valued having the results of an analysis directly linked to relevant source data.

Finally, because many emergency management personnel used social media software infrequently (e.g., only when they were activated into an intelligence role for a large-scale emergency event) it was very important that the software was intuitive. In the pressured environment of a major emergency event, freshly activated intelligence officers need to be able to quickly identify the relevant tools within the software and readily grasp how they work. Thus, one participant commented on the ease with which you could use Signal to create preconfigured searches across multiple social media platforms and another participant noted that the user interface in Geofeedia made it possible to train someone to use it on their own in just five minutes. 


\subsection{Triangulation of multiple sources of information}

Another theme that emerged across the four interviews related to combining multiple sources of information. Intelligence officers used multiple sources of data both to gain greater coverage of information and as a means of validating or assessing the accuracy of findings. All of our interview participants combined information derived from social media monitoring and analysis with traditional sources of intelligence or data. Thus, in the earthquake and tsunami warning centre, duty officers monitored a continuous feed of seismology data in combination with the Twitter feed from the ESA tool and the USGS Twitter Earthquake Detector (Earle et al. 2012).

As well as integrating social media data with other sources of intelligence, all of our interview participants were capturing data from multiple social media platforms. Different social media platforms were seen to have different strengths and weaknesses. For example, Facebook was valuable because it offered detailed information and provided insight into relationship networks. Twitter feeds provided less detail (because of the restricted number of characters) but were good as a source of real-time data. Instagram was useful because it provided access to photographs and the data were more likely to be geo-tagged. Wordpress was useful because it was where "fixated people hang out". Because each platform had different strengths and weakness, interview participants valued social media software (such as Signal and Geofeedia) that allowed them to simultaneously capture information from multiple platforms.

However, the ability to integrate data from multiple sources was valued not only to maximise the breadth of information captured but also to assess the veracity or reliability of social media data. An essential (and timeconsuming) element of an intelligence officer's role lies in evaluating the quality of the information obtained. The police have a formal rating system (the Admiralty code) which involves evaluating both the reliability of the source of the information and confidence in the information itself. They utilise the same process to evaluate data obtained from social media platforms. Before drawing any conclusions from an interesting photo or tweet, an intelligence officer might check previous posts from the same source to see whether he or she had provided accurate information in the past. They might also examine the source's network connections as a means of evaluating their credibility. They might search the web to check whether the photo had been posted previously and cross-check the location of the photo (if it was geo-tagged) against Google Street View to verify that the location looked right. The need to evaluate the quality of the data obtained meant that participants valued software tools that made it easy for them to bring information together from multiple sources.

\section{3. "More eyes on the ground"}

One participant commented that the data provided on social media served as "more eyes on the ground." When members of the community provide information about what they are seeing, hearing and feeling, they can complement the reports coming in from emergency services workers. Community members not only bolster the number of eyes on the ground but they are often privy to different types of information (what people are doing and saying, what resources are available, how the community is coping). Photos posted on social media were especially valuable because of the detail they provided. A photo of a smoke plume or flash flood could inform modeling work by providing more detail as to how a fire is evolving or how quickly a river is rising. Photographs of rain, hailstorms, hailstones or damage to buildings could provide better understanding of the impacts being experienced or factors that might impede recovery efforts. Videos and live streaming were also mentioned as useful, although one participant noted that videos were harder to verify than photos.

\subsection{Geographic and temporal context}

The value of social media data was greatly enhanced when it was geo-tagged or there was some other means of determining the geographic location to which it pertained. In the first instance, understanding where data comes from is vital in order to understand its relevance. Since the emergency services are usually responsible for a specific geographic region, it is useful for them to be able to focus on data that pertains to their area of responsibility. Facebook posts about a protest occurring in Perth do not require attention from emergency services officers in Brisbane. Even once an event is known to be relevant, data that can be geo-located tends to be more valuable than data that cannot. For example, being able to isolate the location of tweets can help in pinpointing the location of someone who is in trouble. Consequently, the value of social media data was greatly reduced because only a very small proportion of these data are geo-tagged. Thus, while participants valued the capability offered by Geofeedia (in particular, the ability to carry out complex searches across multiple platforms within a defined location), the usefulness of this tool was diminished because so much of 
the (non geo-tagged) data potentially available was not captured. The fact that the ESA tool also utilised userprovided location information by geo-coding the user's profile location available as part of the tweet (Power et al. 2014), was seen to be one of its strengths.

Temporal context was also important, although it was mentioned less often than geographic context. The ESA real-time feed was valued not only because it allowed intelligence officers to monitor an event in realtime but also because the timeslider in the word cloud allows users to look back in time and see how the event unfolded. In contrast, Signal only captures data from the point in time when the search query is set up.

\subsection{Information about individuals}

Intelligence officers use terms such as "persons of interest" because some individuals are more important to emergency services personnel than others. For example, a known protestor may be of special interest to police and therefore his or her posts, or posts from other individuals in their network, might provide useful intelligence. In contrast, posts from a journalist might be monitored because they have provided accurate and useful information in the past. One participant commented that he gave greater weight to information when it came from other emergency services officers and military personnel because even if they weren't commenting in their official capacity, their reports tended to be accurate and useful. Thus, the value of social media data was enhanced when it was known to come from a reliable source. Identifying those valuable sources was an important part of the intelligence officer's job. As one participant explained:

$$
\text { ...you spend } 90 \% \text { of your effort excluding the unreliable commentary. [Interviewee 4] }
$$

By honing in on an individual's social media feeds and monitoring them over time, intelligence officers build an understanding of how reliable their information was, who they tended to associate with, where they liked to go and when trouble might occur. All this information was useful when making judgments about where, when and how many emergency service officers might need to be deployed for a particular event.

\subsection{Collaborative tools}

Our participants also valued the ability to be able to communicate their findings with others, quickly and easily. Intelligence officers are conduits of information, their analyses serving to inform other decisionmakers in the chain of response. One participant described using specialised visualisation tools (such as Bottlenose and Tagboard) to build a "storyboard" of their analyses. Being able to quickly communicate the evidence and analysis underlying the key findings was important in the context of social media data since it is still viewed as less trustworthy than more traditional sources of intelligence. Furthermore, decision makers tended to prefer information that was presented in a visual (rather than written) format.

In a major emergency event, intelligence officers will be working in a team so they need tools that allow them to share and combine information. Signal has a feature that allows the user to flag an item for follow-up and write a comment for other users so that they can investigate it further. Finally, since intelligence sometimes needed to be shared between teams working in different organisations (or even with Virtual Operations Support Teams (VOSTs) working within the broader community), one participant sought a common operating picture to underpin social media monitoring and analysis, with shared information management system tools, protocols and architectures.

\subsection{Cost}

Finally, the cost of software was brought up in the interviews. Currently, there is no one software tool that meets all the needs of emergency services organisations. However, the cost of holding multiple licenses for multiple software tools was seen to be a barrier to greater use of social media analytics. There were two issues underlying this problem. First, the need for access to social media monitoring and analysis software varied greatly over time. Ongoing use was relatively low but increased substantially when a major emergency event took place. One participant mentioned that it was helpful to have a pricing model that varied according to level of use, since the cost of multiple licenses could be justified in the event of an emergency but could not be justified on an ongoing basis. Second, there was a lack of consistency in the tools and protocols for social media monitoring and analysis, not only across organisations but even within one emergency management organisation. Not only was it expensive to be supporting multiple software tools but it made cross-agency sharing of information more difficult. For this reason, one participant argued that software should be developed to meet the needs of multiple users rather than being tailored to the unique needs of one emergency services group. 


\section{IMPROVING SOCIAL MEDIA TOOLS}

After exploring the way in which participants currently used social media monitoring and analysis tools to capture intelligence for emergency management, we sought their feedback on how these tools might be improved. Our participants were all using more than one social media monitoring and analysis tools because:

...there's no one tool that does everything that you need. [Interviewee 1]

We therefore asked: "How could social media analytics tools be improved to better meet your organisation's information needs?" Below, we describe how participants responded to this question.

\subsection{Source evaluation}

Most social media users are not known to emergency services organisations and considerable time has to be invested in determining (a) whether the source of the content is a real individual and if so, (b) whether they can be trusted to provide reliable information. Participants suggested that it would be useful to automate some of the elements of this task. One suggested providing a 'source evaluation' metric based on characteristics such as: does the individual have profiles on multiple platforms, how long have the profiles been active; and how many followers or friends do they have. Another suggestion was to differentiate the comments of a direct observer (which are most valuable to emergency services) from retweets or comments by other social media users who are expressing sympathy. Another suggestion was to incorporate some form of "intent" analysis (analogous to sentiment analysis) that differentiates social media content that was just "venting" from social media content that was indicative of a real intent to cause harm.

\subsection{Ability to follow individuals}

As noted above, certain individuals are more important to intelligence officers, either as a "person of interest" or as a reliable source of information. For this reason, it would be useful to have a "follow users" tool that monitors content from certain users across multiple platforms. As one participant explained:

We have people that we know post information regularly in an open source forum (and groups as well) and they can number in the 100s and we want to know what they are posting... Collecting that information is very difficult.... We essentially would like a newsfeed... Are they saying that they are going to have an unplanned protest somewhere, are they saying they want to go and attack a dignitary because of their views on anti-war...? [Interviewee 3]

Some tools partially have this functionality, however, our interview participants wanted to have multiple lists of users who they could follow for different reasons (e.g., you might follow one group of people to stay abreast of anti-nuclear protests and a very different group of people to inform child safety services). They also needed to be able to modify the list when they discovered new commentators or persons of interest.

\subsection{Geographic representation of search results}

Given that emergency services managers tended to prefer information in a visual format, three of our participants suggested that it would be useful to summarise social media content using a map format. The map would provide a high-level view on the geographic spread of comments and the frequency or intensity of that commentary, relative to baseline or normal commentary, thus quickly identifying those locations where the greatest impact is being experienced. One participant wanted to be able to project a map of Australia in the incident room with a bar chart coming out of those locations where there was an increase in the use of disaster-related terminology. Another participant described the earthquake map produced by Geonet (in New Zealand) which depicts where "felt" reports are coming from, how many reports are being received at each location and the intensity of the event being experienced at each location. One suggestion related to the ESA tool in particular: it would be useful if the word cloud or alerts could be tailored for specific regions of interest (e.g., policing districts, or states and territories).

\subsection{Real-time feed across multiple platforms}

Participants also commented on the value of being able to combine real-time feed functionality with data capture from multiple platforms. The changing word cloud in the ESA tool was seen to be a useful way of depicting real time trends in social media content. Possibly a map of Australia could serve the same function. However, currently the ESA tool only captures content from the Twitter platform and participants wanted to be able to have a constantly updating feed of aggregated social media content, with the capability to drill down into results from specific platforms. Instagram was identified as particularly useful because users post 
lots of photos on this platform. However, ideally participants wanted to be able to capture data from all of the platforms used within their communities of interest. Relevant platforms included Facebook, Twitter, Instagram, Sina Weibo and Vkontakt (widely used by Eastern Europeans).

\subsection{Flexibility to modify searches}

Finally, one participant also commented on the need to be able to modify searches as new information was gained. Complex search queries required significant time and effort to set up. After examining the search results, intelligence officers might realise that a certain term needed to be added or excluded. Sometimes they might want to include a new set of content or combine the results of two sets of queries. However, in the current software, the structure of the queries was so complex that such modification was not always possible. The ability to modify searches quickly was important in this intelligence officer's line of work.

\section{CONCLUSIONS}

Our findings were based on four interviews with subject matter experts in three Australian emergency management organisations. Our original intention was to continue with more interviews however this has not occurred. From these few sample interactions we gathered enough evidence to satisfy ourselves of the major factors required to both improve the existing ESA tool and to inform our research direction in this space. Note that the role an agency plays in a disaster or crisis situation, in addition to the type of disaster, may determine the type of information needed and available, thus our findings may not represent the full range of needs in the emergency management space.

The information provided by our participants provides direction for the ongoing development of social media monitoring and analysis tools. At CSIRO, we are carrying out further refinement and improvement of the ESA tool based on the findings from these interviews. Specifically, we have revised the user interfaces, introduced a consistent layout for the common components, simplified the mechanism to define search queries and improved some of the back end system features to make the user interfaces more responsive. These changes reflect most of the findings discovered from these interviews, however there is still some further work that could be done to improve the ESA system. For example the integration of new social media platforms, improved geo-coding of social media content and the automated estimation of the reliability of the information found. These tasks form part of our ongoing research agenda.

\section{ACKNOWLEDGEMENTS}

We are grateful to the four individuals who took part in the interviews and provided the insights we report in this paper. There have been a number of collaborating agencies supporting ESA: the Attorney-General's Department, Geoscience Australia, the NSW Rural Fire Service, the Queensland Department of Community Services, the Queensland Police Service and the Reserve Bank of Australia. The authors thank the contributions of our CSIRO colleagues who have worked on ESA: Mark Cameron, John Colton, Sarvnaz Karimi, Andrew Lampert, John Lingad, Bella Robinson, Peter Marendy, Saguna, Brooke Smith, Gavin Walker, Allan Yin and Jie Yin. Bella, Mark and Robert developed most of the ESA software. Mark Cameron helped construct the interview questions.

\section{REFERENCES}

Department of Homeland Security (DHS). Using Social Media for Enhanced Situational Awareness and Decision Support. June 2014.

Earle PS, Bowden DC, and Guy M. Twitter earthquake detection: earthquake monitoring in a social world. Annals of GeoPhysics, 54(6):708-715, 2012.

Lindsay BR. Social Media and Disasters: Current Uses, Future Options, and Policy Considerations. Analyst in American National Government. Sep 2011.

Plotnick L, Hiltz SR, Kushma J, Tapia A. Red Tape: Attitudes and Issues Related to Use of Social Media by U.S. County-Level Emergency Managers. International Conference on Information Systems for Crisis Response and Management (ISCRAM). Kristiansand, Norway. May 2015.

Power R, Robinson B, Colton J, Cameron M (2014) Emergency Situation Awareness: Twitter Case Studies. ISCRAM-med, Toulouse, France, October 2014. pp. 218-231.

Tapia A, Moore KA, Johnson NJ. Beyond the Trustworthy Tweet: A Deeper Understanding of Microblogged Data Use by Disaster Response and Humanitarian Relief Organizations. International Conference on Information Systems for Crisis Response and Management. Baden-Baden, Germany, May 2013. 